\title{
Progress in the Diagnosis and Treatment of COVID-19 in Children: A Review
}

\author{
Libo Wang' \\ Gan $\mathrm{Li}^{\prime}$ \\ Chang Yuan' \\ Yuele Yang' \\ Gongxia Ling' \\ Jinyu Zheng' \\ Yiyang Zhou' \\ Tianlei Zhang' \\ Wei $\operatorname{Lin}^{2}$ \\ Zhenlang Lin $\mathbb{D}^{2}$ \\ 'Department of Clinical Medicine, \\ Wenzhou Medical University, Wenzhou, \\ 325000, Zhejiang, People's Republic of \\ China; ${ }^{2}$ Department of Pediatrics, The \\ Second Affiliated Hospital and Yuying \\ Children's Hospital of Wenzhou Medical \\ University, Wenzhou, 325000, Zhejiang, \\ People's Republic of China
}

Correspondence: Wei Lin; Zhenlang Lin Email linweilII0@I63.com; Izlprof2020@I63.com

\begin{abstract}
Coronavirus disease 2019 (COVID-19) has been circulating in many countries around the world, characterized by long incubation period, strong infectivity, strong variability, high population susceptibility and diversified transmission methods. Its causative agent is severe acute respiratory syndrome coronavirus 2 (SARS-CoV-2). Compared with adult patients, the clinical manifestations of COVID-19 in children are often dominated by mild or asymptomatic infections, but children are also important virus carriers and play an important role in the transmission of the virus. In addition, some children will show excessive inflammatory response and experience serious complications such as multisystem inflammatory syndrome in children (MIS-C). At present, the research on COVID-19 in children is still imperfect. This article will review epidemiological characteristics, the mechanism of action, variant characteristics, clinical manifestations, auxiliary examinations and treatment of children with COVID-19, in order to provide help for the diagnosis, treatment and research of children with COVID-19.
\end{abstract}

Keywords: children, COVID-19, mutation, clinical manifestations, treatment

\section{Introduction}

SARS-CoV-2 was identified in Wuhan, China in December 2019. ${ }^{1}$ The disease caused by this virus is called COVID-19. COVID-19 is highly infectious. ${ }^{2}$ It has now developed into a global pandemic, affecting more than 214 countries and regions around the world. As of July 2021, the cumulative number of confirmed cases worldwide has exceeded 190 million, and the cumulative number of deaths has exceeded 4 million. COVID-19 has become a major public health threat in the world today. However, even with the emergence of a global pandemic, causing serious global harm, current research on COVID-19 is still imperfect, especially in pediatric groups.

Compared with adult patients, pediatric patients have a smaller number, lower incidence, milder symptoms, and lower mortality (about $0-0.2 \%$ ), better prognosis. ${ }^{3-5}$ In pediatric infected individuals, the incidence of common symptoms with COVID-19 was low. Among them, 59.9\% (80\% in adults) had fever; $55.9 \%$ (84\% in adults) had cough; $20 \%$ (38.4\% in adults) had runny nose. ${ }^{6}$ Currently, there is increasing evidence that individuals in the pre-symptomatic phase carry a large number of viruses with a greater risk of transmission than those in the symptomatic phase, ${ }^{7,8}$ while asymptomatic infected individuals have also been demonstrated to play an important role in the transmission of the virus. ${ }^{9}$ This means that children with COVID-19 who have mild symptoms and few symptoms are more likely to have transmission of the virus due to misdiagnosis and missed diagnosis. ${ }^{10}$ 
Existing studies show that approximately 5\% are infected SARS-CoV-2 Children can become critically ill or critically ill COVID-19, ${ }^{11}$ some children will show excessive inflammatory response and experience MISC. ${ }^{12,13}$ Children with MIS-C are characterized by persistent fever, systemic excessive inflammation, and multiple organ involvement, and many have severe gastrointestinal symptoms as well as symptoms similar to toxic shock syndrome (TSS) such as cardiogenic shock and hypotension, most of which are severe and require pediatric intensive care unit care. ${ }^{14}$ Therefore, deepening the understanding of children with SARS-CoV-2 infection, improving the detection rate of children with COVID-19, and rapidly identifying and treating children with critical symptoms such as MIS-C are important tasks in the current world. This article reviews the progress in the epidemiological characteristics, mechanism of action, variation characteristics, clinical symptoms, auxiliary examination and treatment of COVID-19 in children, with a view to providing help for the diagnosis, treatment and research of children with COVID-19.

\section{Epidemiological Characteristics of COVID-I 9 in Children}

Children are infected mainly through contact with those infected with SARS-CoV-2. The incubation period of the virus can be as long as 24 days. ${ }^{15}$ The virus is mainly spread through respiratory droplets and close contact. ${ }^{3}$ Most children with COVID-19 can excrete the virus through feces, and aerosols or contact with body fluids may also lead to infection in children when feces and urine cause environmental pollution. ${ }^{3,16}$ At the same time, there are reports showing that viruses can be cultivated from wastewater samples, which suggests a possible water transmission route. ${ }^{17}$ Starting from the newborn, children of all ages are likely to be infected with COVID-19. ${ }^{18}$ Existing data show that the main source of infection for children is SARS-CoV-2-positive adults living in the family. ${ }^{19}$ Therefore, timely isolation of adults with a history of epidemiological exposure in the family helps protect children from infection. ${ }^{20}$ In general, compared with adults, children are less likely to transmit SARS$\mathrm{CoV}-2,{ }^{21}$ and children are less susceptible to SARS-CoV -2 with longer incubation period and viral excretion time in feces. ${ }^{17}$ Children have fewer outdoor activities and fewer international travels, and the virus infection rate may be reduced accordingly. ${ }^{22}$ Data also show that children under 5 years of age are less likely to be infected with SARS-CoV-2 than children over 5 years of age, though there is no clear reason for this finding. ${ }^{23}$ At present, the effect of caesarean section on the vertical transmission of COVID-19 is not clear. ${ }^{24}$ Cases of coinfection with SARS-CoV-2 in newborns born to mothers perinatally infected with SARS-CoV-2 are rare. ${ }^{25}$ However, because mothers who have been infected with SARSCoV-2 at the time of delivery are still infectious, newborns are still at risk of contracting the virus from their mothers. ${ }^{26}$ In order to protect newborns from SARS-CoV -2 , perinatal clinicians also need to emphasize the importance of focusing on infection control and preventive measures in the family to SARS-CoV-2-infected mothers, requiring mothers and other nursing staff to meet noninfectious indicators. ${ }^{26}$

Children are generally susceptible to the SARS-CoV $-2{ }^{18}$ However, the severity of disease after infection is lower than in adults. ${ }^{21}$ In China, children account for approximately only $2 \%$ of all COVID-19 infection cases. ${ }^{27}$ Children with underlying diseases (such as type 1 diabetes, congenital abnormalities of heart and circulation, obesity, essential hypertension, epilepsy, neuropsychiatric disorders, asthma, malnutrition, Down syndrome, etc.) are at risk of developing severe cases. ${ }^{28-30}$ Data suggest that children with complex chronic diseases are three times more likely to have severe disease than children without chronic diseases. ${ }^{31}$ Immunocompromised children have a 2.89 -fold increased risk of contracting SARS-CoV-2. ${ }^{32}$ On the other hand, as children age, the risk posed by a history of malnutrition to children with severe COVID-19 decreases only slightly and is highest in younger children (under 5 years of age). ${ }^{29}$ At the same time, the length of hospital stay was significantly prolonged in children with other viral (such as adenovirus, rhinovirus, enterovirus, etc.) infections. ${ }^{33}$

\section{Pathogenesis of COVID-19 in Children}

\section{Mechanism of Cell Invasion by SARS-CoV-2}

SARS-CoV-2 is one of the types of human coronaviruses and belongs to the Beta coronavirus genus. SARS-CoV-2 is mainly composed of membrane, envelope, nucleocapsid and spike glycoprotein (S protein), which plays an important role in the packaging of viral RNA genome and the process of viral infection. ${ }^{34}$ SARS-CoV-2 recognizes cells through the $\mathrm{S}$ protein on its surface and enters cells after 
binding to angiotensin converting enzyme 2 (ACE-2) of cells. Therefore, the body's sensitivity to infection is related to the number of ACE-2. ${ }^{22,34} \mathrm{ACE} 2$ in pediatric individuals is mainly located in type II alveolar cells and can also be expressed in nasal mucosa, upper respiratory tract, heart, kidney and enterocytes. ${ }^{35}$ Therefore, children can present with systemic symptoms of viral infection. ${ }^{35}$ At the same time, ACE- 2 can process angiotensin- 2 into angiotensin-1, 3-7, resulting in vasoconstriction, leukocyte migration, decreased expression of inflammatory cytokines, and activation of fibrinogen, thereby counteracting part of the inflammatory effect. ${ }^{36}$ ACE-2, while showing a bidirectional regulatory role during SARS-CoV-2 infection in children, is more evidence that highly expressed ACE-2 expression can protect patients with lung injury from disease exacerbation. ${ }^{10,37}$ After infection of host cells with SARS-CoV-2, viral RNA is released into the cytoplasm and uses cellular mechanisms for replication, translation. ${ }^{35}$ Newly synthesized viral proteins and RNA genomes bind in the endoplasmic reticulum and Golgi apparatus and release cells through exosomes, ${ }^{35}$ which continuously infect new cells and cause severe tissue damage. In addition, it has been shown that transmembrane serine proteinase-2 (TMPRSS2) can promote $\mathrm{S}$ protein association with ACE-2 and facilitate SARSCoV-2 entry into host cells. ${ }^{38}$ And the androgen receptor, as the transcription promoter of TMPRSS2, can increase the expression of the TMPRSS2 gene. ${ }^{38}$ Therefore, body androgen levels can regulate viral infection through the TMPRSS2/S protein. This may also be the reason why male children have higher admission rates in the Pediatric Intensive Care Unit. ${ }^{13}$ CD147 expressed on the cell membrane surface can be used as an alternative receptor for SARS-COV-2, allowing SARS-COV-2 to invade ACE2deficient $\mathrm{T}$ cells, resulting in SARS-COV-2 infection in children, which may provide research direction for the specific treatment of children with SARS-COV-2 infection. ${ }^{39}$

\section{Immune Damage Caused by SARS-CoV-2 Infection}

Children infected with SARS-CoV-2 will produce corresponding immunopathological responses, involving innate immunity (the role of type I interferon (IFN) and proinflammatory cytokines) and adaptive immunity (the production of neutralizing antibodies against virus-specific antigens by B cells plays a key role in the clearance of
SARS-CoV-2) as well as autoimmunity. ${ }^{40}$ The immune response is bidirectionally regulated after SARS-CoV-2 infection, and its outcome is affected by the degree of immune cell activation and cytokine imbalance. ${ }^{41}$ In addition, host-specific factors also determine to some extent whether an individual develops severe disease. ${ }^{40}$

Studies have found that COVID-19 patients have immunological manifestations such as lymphopenia, antibodydependent enhancement (ADE), and reduced or delayed type IFN. Decreased lymphocytes in children mainly include $\mathrm{CD} 8{ }^{+} \mathrm{T}$ cells, $\mathrm{CD} 4{ }^{+} \mathrm{T}$ cells and natural killer cells (NK). ${ }^{42}$ SARS-CoV-2 may directly infect $T$ cells via ACE2 expressed on T cells, leading to T-cell death, and may also contribute to lymphocyte dysfunction by destroying the secondary lymphoid tissue of the spleen and lymph nodes. ${ }^{43,44}$ In addition, animal models suggest that lymphopenia may be attributed to an increase in the number of regulatory $\mathrm{T}$ cells. ${ }^{45} \mathrm{~B}$ cells are now known to protect against SARS-CoV-2 infection by producing specific antibodies to block ACE2 receptors to prevent viral entry. ${ }^{46}$ However, it has been shown that the severity of COVID19 is associated with increased IgG reactivity. ${ }^{47}$ This may be related to the $\mathrm{ADE}$, the presence of specific antibodies in humans that can bind to SARS-CoV-2 virus and, through their $\mathrm{Fc}$ segment, bind to certain FcR-expressing cells thereby mediating viral entry into these cells and enhancing viral infectivity. ${ }^{48}$ Interferons are an important part of antiviral immunity, in which type I IFNs (IFN- $\alpha$ and IFN- $\beta$ ) are widely expressed in humans. Viral infection can induce IFN production, which in turn triggers the transcription of IFNstimulated genes, which have a variety of antiviral functions. The type I IFN response has been found to be severely impaired in critically ill and critically ill COVID-19 patients (as indicated by IFN- $\beta$ deletion and low IFN- $\alpha$ expression, low activity), which is linked to sustained blood viral load and exacerbated inflammatory response. ${ }^{49}$ Therefore, we should monitor the immune status of SARS-CoV-2 infected children and interfere promptly to avoid pathological damage caused by immune imbalance.

\section{Serious Complications in Pediatric Infected Individuals - MIS-C}

Overall, children with COVID-19 have milder symptoms and a good prognosis relative to adults. On the one hand, children have less ACE2 receptor expression in respiratory cells and some are undifferentiated and mature, so the risk of SARS-CoV-2 infection is low. ${ }^{50,51}$ On the other 
hand, there is a phenomenon of elevated physiological lymphocyte levels, particularly NK cell levels, in the blood of children, and this phenomenon persists after SARS-Cov-2 infection. ${ }^{10}$ Therefore, pediatric patients are less affected by lymphopenia and decreased white blood cell count. ${ }^{22,52}$ In addition, compared with adults, children are less likely to experience uncontrolled immune activation, less inflammatory cytokine (eg, Interleukin-6 (IL-6)) production, and more anti-inflammatory cytokine (eg, Interleukin-10 (IL-10)) production. ${ }^{10}$ Therefore, children have mild symptoms and a good prognosis after viral infection.

Although the prevalence and severity of COVID-19 are low in children, some pediatric patients develop a serious complication similar to Kawasaki disease or TSS, namely MIS-C, and the peak of this case often occurs weeks after the peak of COVID-19. ${ }^{53,54}$ MIS-C cases often present with positive SARS-Cov-2 antibodies and negative RTPCR results, so MIS-C is considered to be an immunemediated post-infectious syndrome. MIS-C may be explained by an abnormal immune or inflammatory response triggered by SARS-CoV-2 infection. ${ }^{55}$ The ratio of neutrophils to lymphocytes can be used as an inflammatory marker to assess the condition of COVID-19 patients with MIS-C. ${ }^{52}$ When the immune system is activated to a certain degree or even out of control, there is an extreme immune attack on the host. When SARS-CoV-2 further invades children, it prompts the human body to secrete cytokines such as interleukin-8, IL-6, IL-10, and tumor necrosis factor- $\alpha$ and produce a cytokine storm, which leads to an excessive inflammatory response and destroys blood vessels, causing MIS-C. ${ }^{56}$ Vascular endothelial injury and vasculitis are important lesions in MIS-C and may also be induced by immune complexes. ${ }^{57}$ $\mathrm{S}$ protein-IgG immune complexes highly expressed in children with MIS-C cause inflammatory responses and vascular injury through Fc $\gamma$ receptor signaling, neutrophil activation and phagocytosis. ${ }^{58,59}$ After global autoantibody screening of children with MIS-C, autoantibodies were found to bind to proteins involved in immune cell signaling as well as structural proteins in the heart and blood vessels. ${ }^{60}$ This suggests that autoimmunity may be greatly involved in the pathological progression of MIS-C such as myocarditis and vasculitis. In addition, the efficacy of intravenous immunoglobulin (IVIG) also supports the hypothesis that autoimmune reactions are involved in the pathogenesis of MIS-C. ${ }^{61}$

\section{The Mutation of the New Coronavirus Types of Mutations}

Studies have shown that over time, the SARS-COV-2 produces mutations, and as the accumulation of the number of mutations, eventually certain mutational features will dominate, allowing mutational viral outbreaks. ${ }^{34}$ So far, there are 4 globally recognized variants: Alpha or pedigree B.1.1.7 (UK), Beta or pedigree B.1.351 (South Africa), Gamma or pedigree P.1 (Japan/Brazil), and Delta or pedigree B.1.351 (South Africa) pedigree B.1.617.2 (India). ${ }^{62}$ The $\mathrm{S}$ protein contains two subunits: $\mathrm{S} 1$, which contains the receptor binding domain (RBD), and S2, which mediates virus-host cell fusion. ${ }^{63}$ The most common mutation in the viral genome is the substitution of D614G (Asp 614 $\rightarrow$ Gly) in S1, which increases the affinity of the virus to the ACE2 receptor, leading to increased host susceptibility and increased transmission. ${ }^{63,64}$

\section{Significance of Mutations}

Viruses obtain more effective functions such as virus replication, transmission and evasion of host defense functions through mutation and natural selection. ${ }^{63}$ Among the people infected with the mutant strain, about $80 \%$ have only mild symptoms, these patients are ignored by early clinical screening because they have only mild symptoms but no typical early clinical symptoms such as fever, cough and upper respiratory tract infection, and about $20 \%$ with severe symptoms of "cytokine storm". ${ }^{65}$ Due to the recognition and binding of $\mathrm{S}$ protein, $\mathrm{S}$ protein and its $\mathrm{RBD}$ are expected to become vaccine targets for SARS-CoV-2. ${ }^{65}$ The neutralizing antibodies in serum mainly target RBD domain motifs, in which mutations will significantly affect the neutralization of antibodies. ${ }^{65}$ Major viral mutations reduced the neutralization sensitivity of recently vaccinated individuals to convalescent plasma antibodies. ${ }^{63}$ Investigating emerging, rapidly spreading variants and ongoing assessment of the phenotype for neutralization of post-vaccination sera should be a high priority for monitoring the impact of vaccine efficacy. ${ }^{63}$ There are data showing that SARS-CoV-2 mutants in some patients are 5-fold less sensitive to neutralization by convalescent plasma antibodies. ${ }^{63}$ In addition, the D614G mutation did not affect the virus replication or viral particle infectivity in Vero E6 cells, but the infectivity and stability of SARS-CoV-2 produced by the human lung cell line increased after the mutation, while the D614G mutation had the potential to change the conformation of the spike protein, thereby 
affecting the neutralization of monoclonal antibodies in an epitope-specific manner. ${ }^{66}$ More and more evidence show that immune escape mutations and a variety of fastspreading mutations will appear in long-term SARS-CoV -2 infections. ${ }^{63}$ We should focus on these mutations and variants in these infections and study them accordingly. ${ }^{63}$

Within 5 weeks after the first identification of the Delta variant, the main virus variant in Mesa County, Colorado is the Delta variant, which is now the main variant in the United States. ${ }^{67}$ The intensive care unit admissions and case fatality rate in this county suggests that infection with the Delta variant may cause more serious results. ${ }^{67}$ Although the vaccine can reduce the transmission potential of SARS-CoV-2 and reduce the risk of virus infection in humans, the Delta variant affects the effect of the vaccine and may increase the risk of virus transmission. ${ }^{68}$ Although the specific mechanism of action of the variants on the vaccine has not been elucidated, more importantly, the ability of antibodies to neutralize the Delta variant is reduced compared to the Alpha variant, and the Delta variant is more transmissible and attenuates 10 -fold the immune protection of the human body. ${ }^{69}$ But in most cases, the level of neutralizing antibodies induced by the vaccine is sufficient to protect them. ${ }^{69}$ In order to prevent the further emergence of immune escape variants, a comprehensive global vaccination and prevention strategy is needed to reduce the spread of SARS-CoV- $2,{ }^{63}$ the safest and most controllable way to prevent COVID-19. ${ }^{35}$ Where children have frequent contact with grandparents, measures to vaccinate children are beneficial in reducing the possibility of transmission of SARS-CoV-2 to the elderly. ${ }^{70}$ On the other hand, children's vaccination facilitates their return to school and return to normal life. ${ }^{71}$ Given the evolutionary nature of the SARS-CoV-2 genome, drug and vaccine developers should continue to be alert to the emergence of new variants or substrains of the virus. ${ }^{64}$ Recent data show that some vaccines have weaker resistance to B.1.351 variants, which requires further research. ${ }^{63}$ However, the reduced sensitivity of antibodies to new variants does not essentially prove that the vaccine is less effective. ${ }^{63}$

\section{Clinical Characteristics of COVID- I 9 in Children \\ Clinical Manifestations}

Mild or asymptomatic infection is the main clinical manifestation in pediatric patients, mainly manifested as fever, sore throat, shortness of breath and pneumonia. ${ }^{72}$ Compared with adults, vomiting and diarrhea are more common in pediatric patients, while the incidence of fever and pulmonary symptoms is lower, ${ }^{22}$ and anosmia and taste are less common. ${ }^{73}$ Children with cancer, children undergoing hematopoietic cell transplantation, children undergoing solid organ transplantation and other children taking immunosuppressive agents mostly present with asymptomatic or mild gastrointestinal symptoms due to low immune function and weak immune response after infection with COVID-19, ${ }^{32,74}$ with a low risk of death. ${ }^{75}$ The frequency of dyspnea and mortality in immunocompromised adults hospitalized may be due to their incidence of complications (such as hypertension, cardiovascular disease and diabetes). ${ }^{32}$ Children have relatively fewer neurological complications after infection with SARSCoV-2 compared with adults. ${ }^{76}$ Neurological symptoms are mainly non-specific symptoms such as headaches, and the incidence of severe symptoms and specific complications is only about $1 \% .^{76}$ This also causes respiratory Children with insignificant symptoms are easily misdiagnosed and missed. ${ }^{77}$ Children with severe infections may develop acute respiratory distress syndrome, septic shock, metabolic acidosis and coagulopathy. ${ }^{22}$ The results of the study showed that the hospitalization and intensive care time, lymphocytosis, and abnormal CT findings (groundglass opacities and consolidation) of children in the critically ill group were higher than those in non-critically ill children, which are the most reliable factors related to the severity of COVID-19. ${ }^{78}$

Some children with progressive disease may develop serious complications MIS-C. The complications are prominent in cardiovascular lesions with clinical manifestations similar to macrophage activation syndrome, symptoms of staphylococcal and streptococcal TSS, sepsis. ${ }^{79}$ Other systems also affected include the respiratory system (eg, manifestations of respiratory distress), urinary system (eg, anuria, oliguria), hematologic (eg, active bleeding and thrombosis), digestive system (eg, diarrhoea, liver involvement), nervous system (eg, irritability, confusion), etc. ${ }^{13}$ Compared with children without MIS-C, children with MIS-C had less sore throat and respiratory symptoms, inflammation (such as fever and rash) and gastrointestinal (such as vomiting and diarrhea) symptoms. ${ }^{72}$ In addition, neurological complications that are rarely seen in children infected with SARS-CoV-2 are particularly likely to occur in SARS-CoV-2 infected children with MIS-C, among which encephalopathy is more 
common, sometimes accompanied by transient symptomatic seizures, but without long-term neurological sequelae. $^{76}$ Therefore, the possibility of being one of the clinical manifestations of MIS-C should be considered in any child presenting with primary neurological symptoms, accompanied by fever and mild or no respiratory symptoms in an endemic area of COVID-19. In addition, serum albumin levels ${ }^{13}$ and croup $^{80}$ can also be closely monitored as predictive indicators of MIS-C. Due to most children with MIS-C have mild or no symptoms when initially infected with COVID-19, and high vigilance is needed for MIS-C. ${ }^{81}$

\section{Imaging Manifestations}

Chest computed Tomography (CT) can be used for screening diagnosis of COVID-19 infection, showing disease progression, and has great promise in adult symptom screening applications. ${ }^{82,83}$ However, because the clinical symptoms of children with COVID-19 are relatively mild, difficult to detect by $\mathrm{CT}$, and CT will produce high-dose radiation, the necessity of chest $\mathrm{CT}$ should be proposed by pediatricians according to clinical needs. ${ }^{83}$ The most common CT abnormalities in children with COVID-19 pneumonia are peripheral and/or subpleural mixed ground-glass opacities and consolidation. ${ }^{84}$ In more than half of the children with abnormal CT examinations, the "halo" sign is seen, that is, focal consolidation, surrounded by ground glass shadows, and bronchial wall thickening and peribronchovascular opacity are more frequent in children than in adults. ${ }^{84}$ If multiple lobular lesions appear in the lungs and white lungs of children, it indicates a high inflammatory response. ${ }^{82}$ After active treatment of children, lung lesions can be absorbed to varying degrees. ${ }^{82}$ Chest X-ray and CT findings of infants are atypical, and still need to further research and summary. ${ }^{82}$

$86.4 \%$ of pediatric patients with COVID-19 infection can be correctly diagnosed by lung ultrasound (LUS). ${ }^{85}$ In the absence of reliable, highly sensitive diagnostic tests or when there are too many requests for nasal swab, the LUS can be used to diagnose children with suspected COVID19 and persistent cough, because ultrasound can highlight the interstitial lung lesions caused by COVID-19 and can be used to strengthen the diagnostic ability in children with suspected COVID-19 pneumonia. $^{86}$ Standardized LUS protocols combine ultrasound probability patterns with clinical phenotypes, and both LUS high likelihood patterns and LUS moderate likelihood patterns are very sensitive to the exclusion of interstitial pneumonia, which increases in sensitivity when the child's clinical symptoms worsen. $^{87}$ During the COVID-19 pandemic, LUS can quickly identify children with or without COVID-19 pneumonia infection, which helps clinicians to quickly guide the management of children to use hospital resources more efficiently. ${ }^{87}$ On the other hand, On the other hand, CT, although the gold standard in the assessment of pneumothorax, is limited by the inconvenience of transporting critically ill patients and their high dose of radiation. ${ }^{88}$ Nursing lung ultrasound can reduce the exposure of ionizing radiation, so it has become an effective auxiliary means for the diagnosis of pneumothorax in the postdiagnosis follow-up of children with SARS-CoV-2. ${ }^{88}$ However, there are still reports showing that LUS incorrectly diagnosed COVID-19 in 45\% of uninfected individuals, so LUS needs to be used reasonably in clinic. ${ }^{85}$

\section{Laboratory Auxiliary Examination}

Prompt diagnosis of children infected with SARS-CoV-2 is not only important for individuals, but also essential to reduce the spread of the virus during the pandemic. ${ }^{89}$ Abnormal results of COVID-19 laboratory tests in children are less common than in adults. ${ }^{90}$ Cases of lymphopenia are very common in adults with COVID-19, while only $5.5 \%$ of children present with this test result. ${ }^{90}$ Children are less likely to have leukopenia, and C-reactive protein (CRP), Erythrocyte sedimentation rate (ESR), alanine aminotransferase levels, lactate dehydrogenase levels, and aspartate aminotransferase levels are not significantly increased, while phosphocreatine isoenzyme levels are often significantly increased..$^{90}$ The level of procalcitonin in most children is normal, and the number of D-dimer and ferritin in the blood of severely ill children increases, and the number of lymphocytes gradually decreases. ${ }^{3}$ The number of neutrophils in children with ordinary COVID-19 can be reduced, but the number of neutrophils in severely ill children is significantly increased. ${ }^{52}$ For example, in children with progressive inflammation, they often present with lymphopenia, hypoalbuminemia, hypertriglyceridemia, and elevated IL6, ESR, lactate dehydrogenase, CRP, fibrinogen, procalcitonin, D-dimer, and serum ferritin. ${ }^{13}$ The levels of bilirubin and liver enzymes can be used to diagnose the severity of childhood diseases. ${ }^{22}$ In short, SARS-COV-2 can cause abnormal hematological parameters in children, which is different from adult examination results. This finding is conducive to early diagnosis and treatment of COVID-19 in children. 


\section{Treatment of COVID- 19 in Children General Treatment}

It is recommended that most children with COVID-19 use supportive care. ${ }^{91}$ Effective oxygen therapy measures can be given under hypoxia, and nasal high-flow oxygen ventilation, non-invasive or invasive mechanical ventilation, prone position ventilation therapy or lung recruitment and extracorporeal membrane oxygenation can be taken, when necessary, according to the child's situation. ${ }^{3}$ For children with high fever, convulsions or epileptic seizures, the corresponding drugs should be used to control the condition first to ensure that the airway is unobstructed before treatment. $^{3}$

\section{Antiviral Treatment}

Antiviral drugs (eg, remdesivir) can be used to treat critically ill or critically ill children (age $\geq 12$ years, weight $\geq$ $40 \mathrm{~kg}$ ) who require oxygen supplementation, or invasive mechanical ventilation or extracorporeal membrane oxygenation. ${ }^{92}$ COVID-19 monoclonal antibody therapy is not recommended for children, but infliximab can effectively treat children with Crohn's disease and prevent the systemic inflammatory response in children. ${ }^{93}$ Other drugs including chloroquine, hydroxychloroquine, azithromycin, or lopinavir/ritonavir are also not recommended for the treatment of COVID-19 in children. ${ }^{92}$ Drug development has focused on targets for viral entry into the host and on viral RNA-dependent RNA polymerases. ${ }^{94}$ Due to the emergence of SARS-CoV-2 variants, effective and broadspectrum antiviral drugs against SARS-CoV-2 and its variants need to be investigated, such as fusion inhibitors targeting the conserved heptapeptide repeat region 1 in the S2 subunit of the S protein. ${ }^{95}$

\section{Traditional Chinese Medicine Treatment}

Traditional Chinese Medicine (TCM) bases on syndrome differentiation and treatment. ${ }^{96}$ Through the analysis of signs and prescriptions, it can prevent COVID-19, which reflects the idea of "prevention and treatment", and has an integrated mechanism of anti-virus, anti-inflammatory, immunomodulatory and organ protection. ${ }^{96}$ It can improve the cure rate, delay the course of the disease, reduce mortality, and treat COVID-19. ${ }^{96}$ In terms of prevention, herbal fragrances, Chinese herbal decoctions, moxibustion, food therapy, Chinese medicine substitutes for tea, and Chinese herbal foot baths can be used, thus cutting off the route of transmission. ${ }^{97,98}$ In terms of TCM treatment,
Chinese medicine decoctions and Chinese patent medicines can be used for treatment, and the corresponding Chinese patent medicines can be used according to the clinical manifestations. ${ }^{97}$ At the same time, acupuncture therapy can regulate the human immune system, and different acupoints are used in different clinical stages. ${ }^{97}$ Acupuncture is expected to become a safe and effective adjuvant therapy. ${ }^{97}$ According to reports, integrated traditional Chinese and western medicine can shorten the treatment time of COVID-19 patients, improve the clinical cure rate, and play a huge role in fighting the COVID-19 pandemic. $^{99}$ Because the disease spectrum of children is different from that of adults, the effectiveness and safety data of TCM in treating children are not comprehensive, and this method needs further study. ${ }^{100}$

\section{Adjuvant Treatment}

Vitamin $\mathrm{C}$ has the potential to control inflammation, but there is still no evidence to prove that vitamin $\mathrm{C}$ supplementation can effectively reduce the risk of SARS-CoV2 infection. ${ }^{101}$ Vitamin D can play a role in preventing or treating SARS-CoV2 infectious diseases by improving the immune response of children to the virus. ${ }^{102}$ Folic acid is also a potential treatment for COVID-19. ${ }^{103}$ In addition, the COVID-19 virus spike protein uses TMPRSS2 to invade the host. ${ }^{104}$ Androgen receptor is the transcription promoter of TMPRSS2. ${ }^{38}$ Therefore, androgen can promote the entry of COVID-19 virus into host cells. TMPRSS2 inhibitors or ACE2 receptor binding competitive inhibitors are the active ingredients to prevent the new coronavirus, so it is possible to treat COVID-19 by inhibiting androgens, while bromhexine, a drug with both favorable and safe properties, gives clinicians a better treatment strategy, and the specific therapy is still under investigation. ${ }^{104}$

\section{Treatment for MIS-C}

Supportive intensive care, immune modulation (steroids and intravenous immunoglobulins), and organ support therapy (including hemodynamic support) are effective in children with MIS-C. ${ }^{55}$ In the pediatric MIS-C treatment consensus, intravenous immunoglobulin is recommended as the first-line treatment, corticosteroids as the second-line drugs, and anticoagulants according to the child's condition. ${ }^{105}$ At present, the most widely recommended is intravenous immunoglobulin drugs, ${ }^{106}$ the drug helps restore the systolic function of the left ventricle. ${ }^{107}$ In addition, the length of hospital stay of 
children is shortened after applying corticosteroids to MIS-C treatment. ${ }^{108}$ For example, low-dose prednisolone can shorten the length of hospital stay in children with moderate to severe COVID-19. ${ }^{109}$ There have been cases of MIS-C that have fully recovered with oral steroid therapy alone. ${ }^{110}$ Another report also indicated that the risk of cardiovascular dysfunction was lower in MIS-C children with cardiac insufficiency treated with IVIG in combination with corticosteroids compared with IVIG treatment alone, ${ }^{111}$ shorter time to recovery of cardiac function. ${ }^{112}$ However, because corticosteroids may slow the rate of viral clearance and increase the risk of infection in children, and the optimal dose and duration of corticosteroid therapy in children with MIS-C have not been clarified. ${ }^{113}$ Therefore, the use of corticosteroids should be used with caution according to the clinical condition of the child.

\section{Treatment of Immunocompromised Children}

Immunocompromised children mainly include: children with congenital immunodeficiency, children with acquired immunodeficiency caused by immunosuppressive therapy and children with immune dysfunction caused by related chronic diseases (such as organ dysfunction or failure or severe inflammatory diseases). ${ }^{114}$ Immunosuppression appears to be a protective factor, making immunocompromised children at lower risk of developing critical illness. ${ }^{115}$ Therefore, a certain dose of immunosuppressive agents should be maintained in children on long-term immunosuppressive therapy to prevent recurrence of underlying diseases. On this basis, attempts at antiviral therapy in immunocompromised children can control viral invasion and improve the prognosis of children. ${ }^{75,115}$ For children who do not respond to antiviral therapy, IVIG shows good efficacy without significant complications for the time being. ${ }^{115}$ Moderate antiinflammatory therapy (such as anti-IL-6 or corticosteroids) may also be tried in children with progressive pneumonia. ${ }^{116}$ In summary, The treatment of immunocompromised children should be decided based on their clinical manifestations. ${ }^{117}$

\section{Other Treatments}

Blood purification therapy can be used to remove inflammatory factors and block the "cytokine storm"; bronchoscopy and lavage can ensure normal airway ventilation and prevent obvious airway obstruction; ${ }^{3}$ the use of convalescent plasma therapy to treat severely ill children is safe and effective, ${ }^{118}$ and there is evidence that this therapy does not increase the risk of thromboembolism in children with COVID-19; ${ }^{119}$ appropriate antibiotics (such as azithromycin) can be used control secondary bacterial infections. ${ }^{120}$

\section{Abbreviations}

COVID-19, coronavirus disease 2019; SARS-CoV-2, severe acute respiratory syndrome coronavirus 2; MIS-C, multisystem inflammatory syndrome in children; TSS, toxic shock syndrome; S protein, spike glycoprotein; ACE-2, angiotensin converting enzyme 2; TMPRSS2, transmembrane protease serine 2; IFN, interferon; ADE, antibody-dependent enhancement; NK, natural killer; IL-6, interleukin-6; IL-10, interleukin-10; CRP, C-reactive protein; ESR, erythrocyte sedimentation rate; RBD, receptor binding domain; CT, computed tomography; LUS, lung ultrasound; TCM, traditional Chinese medicine; IVIG, intravenous immunoglobulin.

\section{Summary}

As a special group, most children with COVID-19 present asymptomatic or with mild symptoms and are less affected in the COVID-19 pandemic, but children are also important virus carriers and may also die due to missed diagnosis and misdiagnosis or combined serious complications. For most children with COVID-19, only general supportive therapy is sufficient. However, for critically ill children with serious complications such as MIS-C or underlying immunocompromised patients, after comprehensive evaluation, rational use of IVIG, steroids and antiviral therapy and other measures can reduce the occurrence of serious hemodynamic complications and improve the prognosis of critically ill children. Current research on children infected with SARSCoV-2 is still imperfect and there are still few available drugs. Clinicians should maintain a high degree of vigilance for children with the risk and potential symptoms of SARSCoV-2 infection, pay attention to the clinical diagnosis, treatment and prognosis of pediatric COVID-19 patients, reduce the risk of virus transmission caused by missed diagnosis, and reduce the mortality of pediatric COVID-19.

\section{Acknowledgment}

Wei Lin and Zhenlang Lin contributed equally to this work and should be considered co- corresponding author. 


\section{Disclosure}

The authors report no conflicts of interest in this work.

\section{References}

1. Huang C, Wang Y, Li X, et al. Clinical features of patients infected with 2019 novel coronavirus in Wuhan, China. Lancet. 2020;395(10223):497-506. doi:10.1016/S0140-6736(20)30183-5

2. Li M, Zhu D, Yang J, et al. Clinical treatment experience in severe and critical COVID-19. Mediators Inflamm. 2021;20 21:9924542. doi:10.1155/2021/9924542

3. Shen KL, Yang YH, Jiang RM, et al. Updated diagnosis, treatment and prevention of COVID-19 in children: experts' consensus statement (condensed version of the second edition). World J Pediatr. 2020;16(3):232-239. doi:10.1007/s12519-020-00362-4

4. Sahi PK, Jhamb U, Dabas A. Pediatric coronavirus disease 2019: clinical features and management. Indian Pediatr. 2021;58 (5):453-460. doi:10.1007/s13312-021-2216-4

5. Girona-Alarcon M, Bobillo-Perez S, Sole-Ribalta A, et al. The different manifestations of COVID-19 in adults and children: a cohort study in an intensive care unit. BMC Infect Dis. 2021;21(1):87. doi:10.1186/s12879-021-05786-5

6. Khera N, Santesmasses D, Kerepesi C, et al. COVID-19 mortality rate in children is U-shaped. Aging. 2021;13(16):19954-19962. doi:10.18632/aging. 203442

7. He X, Lau EHY, Wu P, et al. Temporal dynamics in viral shedding and transmissibility of COVID-19. Nat Med. 2020;26 (5):672-675. doi:10.1038/s41591-020-0869-5

8. Jing QL, Liu MJ, Zhang ZB, et al. Household secondary attack rate of COVID-19 and associated determinants in Guangzhou, China: a retrospective cohort study. Lancet Infect Dis. 2020;20 (10):1141-1150. doi:10.1016/S1473-3099(20)30471-0

9. Vermund SH, Pitzer VE. Asymptomatic transmission and the infection fatality risk for COVID-19: implications for school reopening. Clin Infect Dis. 2021;72(9):1493-1496. doi:10.1093/ cid/ciaa855

10. Massalska MA, Gober HJ. How children are protected from COVID-19? A historical, clinical, and pathophysiological approach to address COVID-19 susceptibility. Front Immunol. 2021;12:646894. doi:10.3389/fimmu.2021.646894

11. Kortz TB, Connolly E, Cohen CL, et al. Diagnosis and acute management of COVID-19 and multisystem inflammatory syndrome in children. Pediatr Emerg Care. 2021;37(10):519-525. doi:10.1097/PEC.0000000000002538

12. Rowley AH. Understanding SARS-CoV-2-related multisystem inflammatory syndrome in children. Nat Rev Immunol. 2020;20 (8):453-454. doi:10.1038/s41577-020-0367-5

13. Haslak F, Barut K, Durak C, et al. Clinical features and outcomes of 76 patients with COVID-19-related multi-system inflammatory syndrome in children. Clin Rheumatol. 2021;40(10):4167-4178. doi:10.1007/s10067-021-05780-x

14. Noval Rivas M, Porritt RA, Cheng MH, et al. COVID-19associated multisystem inflammatory syndrome in children (MIS-C): a novel disease that mimics toxic shock syndrome-The superantigen hypothesis. J Allergy Clin Immunol. 2021;147 (1):57-59. doi:10.1016/j.jaci.2020.10.008

15. Mansourian M, Ghandi Y, Habibi D, et al. COVID-19 infection in children: a systematic review and meta-analysis of clinical features and laboratory findings. Arch Pediatr. 2021;28(3):242-248. doi:10.1016/j.arcped.2020.12.008

16. Cai J, Wang X, Zhao J, et al. Comparison of clinical and epidemiological characteristics of asymptomatic and symptomatic SARS-CoV-2 infection in children. Virol Sin. 2020;35(6): 803-810. doi:10.1007/s12250-020-00312-4
17. Grudlewska-Buda K, Wiktorczyk-Kapischke N, WałeckaZacharska E, et al. SARS-CoV-2-morphology, transmission and diagnosis during pandemic, review with element of meta-analysis. J Clin Med. 2021;10(9):1962. doi:10.3390/jcm10091962

18. Stacevičienè I, Burokienè S, Steponavičienė A, et al. A cross-sectional study of screening for coronavirus disease 2019 (COVID-19) at the pediatric emergency department in Vilnius during the first wave of the pandemic. Eur J Pediatr. 2021;180 (7):2137-2145. doi:10.1007/s00431-021-03999-z

19. Siebach MK, Piedimonte G, Ley SH. COVID-19 in childhood: transmission, clinical presentation, complications and risk factors. Pediatr Pulmonol. 2021;56(6):1342-1356. doi:10.1002/ppul.25344

20. Li X, Rong Y, Zhang P, et al. Differences in clinical features and laboratory results between adults and children with SARS-CoV-2 Infection. Biomed Res Int. 2020;2020:6342598. doi:10.1155/2020/ 6342598

21. Gaythorpe KAM, Bhatia S, Mangal T, et al. Children's role in the COVID-19 pandemic: a systematic review of early surveillance data on susceptibility, severity, and transmissibility. Sci Rep. 2021;11(1):13903. doi:10.1038/s41598-021-92500-9

22. Zare-Zardini H, Soltaninejad H, Ferdosian F, et al. Coronavirus disease 2019 (COVID-19) in children: prevalence, diagnosis, clinical symptoms, and treatment. Int $J$ Gen Med. 2020;13:477-482. doi:10.2147/IJGM.S262098

23. Alharbi M, Kazzaz YM, Hameed T, et al. SARS-CoV-2 infection in children, clinical characteristics, diagnostic findings and therapeutic interventions at a tertiary care center in Riyadh, Saudi Arabia. J Infect Public Health. 2021;14(4):446-453. doi:10.1016/j.jiph.2020.12.034

24. Li X, Sun L, Li T. Updates in neonatal coronavirus disease 2019: what can we learn from detailed case reports?. Mol Med Rep. 2021;23(5). doi:10.3892/mmr.2021.11990

25. Gale C, Quigley MA, Placzek A, et al. Characteristics and outcomes of neonatal SARS-CoV-2 infection in the UK: a prospective national cohort study using active surveillance. Lancet Child Adolesc Health. 2021;5(2):113-121. doi:10.1016/S2352-4642(20)30342-4

26. Kaufman DA, Puopolo KM. Infants born to mothers with COVID-19-making room for rooming-in. JAMA Pediatr. 2021;175(3):240-242. doi:10.1001/jamapediatrics.2020.5100

27. Kumar A, Singh R, Kaur J, et al. Wuhan to world: the COVID-19 pandemic. Front Cell Infect Microbiol. 2021;11:596201. doi:10.3389/fcimb.2021.596201

28. Kantar A, Mazza A, Bonanomi E, et al. COVID-19 and children with Down syndrome: is there any real reason to worry? Two case reports with severe course. BMC Pediatr. 2020;20(1):561. doi:10.1186/s12887-020-02471-5

29. Kurtz A, Grant K, Marano R, et al. Long-term effects of malnutrition on severity of COVID-19. Sci Rep. 2021;11(1):14974. doi:10.1038/s41598-021-94138-z

30. Kompaniyets L, Agathis NT, Nelson JM, et al. Underlying medical conditions associated with severe COVID-19 illness among children. JAMA Netw Open. 2021;4(6):e2111182. doi:10.1001/ jamanetworkopen.2021.11182

31. Schuster JE, de St Maurice A. COVID-19 in children-not just little adults. JAMA Netw Open. 2021;4(6):e2111441. doi:10.1001/ jamanetworkopen.2021.11441

32. Rao SK, Kumar A, Prasad R, et al. Clinical profile and outcome of COVID-19 among immunocompromised children. Indian Pediatr. 2021;58(7):686-687. doi:10.1007/s13312-021-2267-6

33. Karaaslan A, Çetin C, Akın Y, et al. Coinfection in SARS-CoV-2 infected children patients. J Infect Dev Ctries. 2021;15 (6):761-765. doi:10.3855/jidc. 14314

34. Tasakis RN, Samaras G, Jamison A, et al. SARS-CoV-2 variant evolution in the United States: high accumulation of viral mutations over time likely through serial Founder Events and mutational bursts. PLoS One. 2021;16(7):e255169. doi:10.1371/ journal.pone. 0255169 
35. Aygün D, Önal P, Apaydın G, et al. Coronavirus infections in childhood and vaccine studies. Turk Arch Pediatr. 2021;56 (1):10-14.

36. Felsenstein S, Hedrich CM. SARS-CoV-2 infections in children and young people. Clin Immunol. 2020;220:108588. doi:10.1016/ j.clim.2020.108588

37. $\mathrm{Gu} \mathrm{H}, \mathrm{Xie} \mathrm{Z}, \mathrm{Li} \mathrm{T}$, et al. Angiotensin-converting enzyme 2 inhibits lung injury induced by respiratory syncytial virus. Sci Rep. 2016;6:19840. doi:10.1038/srep19840

38. Mohamed MS, Moulin TC, Schiöth HB. Sex differences in COVID-19: the role of androgens in disease severity and progression. Endocrine. 2021;71(1):3-8. doi:10.1007/s12020-02002536-6

39. Wang K, Chen W, Zhang Z, et al. CD147-spike protein is a novel route for SARS-CoV-2 infection to host cells. Signal Transduct Target Ther. 2020;5(1):283. doi:10.1038/s41392-020-00426-x

40. van Eijk LE, Binkhorst M, Bourgonje AR, et al. COVID-19: immunopathology, pathophysiological mechanisms, and treatment options. J Pathol. 2021;254:307-331.

41. Liu Y, Sawalha AH, Lu Q. COVID-19 and autoimmune diseases. Curr Opin Rheumatol. 2021;33(2):155-162. doi:10.1097/BOR.00 00000000000776

42. Tsai SF, Lu KY, Chuang HM, et al. Surviving the Rookie virus, Severe Acute Respiratory Syndrome Coronavirus 2 (SARS-CoV2): the immunopathology of a SARS-CoV2 infection. Cell Transplant. 2021;30:963689721993769. doi:10. 1177/0963689721993769

43. Tan L, Wang Q, Zhang D, et al. Lymphopenia predicts disease severity of COVID-19: a descriptive and predictive study. Signal Transduct Target Ther. 2020;5(1):33. doi:10.1038/s41392-020-0148-4

44. Xu H, Zhong L, Deng J, et al. High expression of ACE2 receptor of 2019-nCoV on the epithelial cells of oral mucosa. Int J Oral Sci. 2020;12(1):8. doi:10.1038/s41368-020-0074-x

45. Zheng H, Li H, Guo L, et al. Virulence and pathogenesis of SARS-CoV-2 infection in rhesus macaques: a nonhuman primate model of COVID-19 progression. PLoS Pathog. 2020;16(11): e1008949. doi:10.1371/journal.ppat.1008949

46. Nielsen SCA, Yang F, Jackson KJL, et al. Human B cell clonal expansion and convergent antibody responses to SARS-CoV-2. Cell Host Microbe. 2020;28(4):516-525.e5. doi:10.1016/j.chom. 2020.09.002

47. Zhang B, Zhou X, Zhu C, et al. Immune phenotyping based on the neutrophil-to-lymphocyte ratio and IgG level predicts disease severity and outcome for patients with COVID-19. Front Mol Biosci. 2020;7:157. doi:10.3389/fmolb.2020.00157

48. Yang L, Xie X, Tu Z, et al. The signal pathways and treatment of cytokine storm in COVID-19. Signal Transduct Target Ther. 2021;6(1):255.

49. Hadjadj J, Yatim N, Barnabei L, et al. Impaired type I interferon activity and inflammatory responses in severe COVID-19 patients. Science. 2020;369(6504):718-724. doi:10.1126/science. abc6027

50. Bunyavanich S, Do A, Vicencio A. Nasal gene expression of angiotensin-converting enzyme 2 in children and adults. JAMA. 2020;323(23):2427-2429. doi:10.1001/jama.2020.8707

51. Rawat M, Chandrasekharan P, Hicar MD, et al. COVID-19 in newborns and infants-low risk of severe disease: silver lining or dark cloud? Am J Perinatol. 2020;37(8):845-849. doi:10.1055/ s-0040-1710512

52. Liu L, She J, Bai Y, et al. SARS-CoV-2 infection: differences in hematological parameters between adults and children. Int $J$ Gen Med. 2021;14:3035-3047. doi:10.2147/IJGM.S313860

53. Verdoni L, Mazza A, Gervasoni A, et al. An outbreak of severe Kawasaki-like disease at the Italian epicentre of the SARS-CoV-2 epidemic: an observational cohort study. Lancet. 2020;395 (10239):1771-1778. doi:10.1016/S0140-6736(20)31103-X
54. Feldstein LR, Rose EB, Horwitz SM, et al. Multisystem inflammatory syndrome in U.S. children and adolescents. $N$ Engl $J$ Med. 2020;383(4):334-346. doi:10.1056/NEJMoa2021680

55. Takia L, Angurana SK, Nallasamy K, et al. Updated management protocol for multisystem inflammatory syndrome in children (MIS-C). J Trop Pediatr. 2021;67(3). doi:10.1093/tropej/fmab071

56. Diorio C, Henrickson SE, Vella LA, et al. Multisystem inflammatory syndrome in children and COVID-19 are distinct presentations of SARS-CoV-2. J Clin Invest. 2020;130(11):5967-5975. doi:10.1172/JCI140970

57. McMurray JC, May JW, Cunningham MW, et al. Multisystem inflammatory syndrome in children (MIS-C), a post-viral myocarditis and systemic vasculitis-a critical review of its pathogenesis and treatment. Front Pediatr. 2020;8:626182. doi:10.3389/ fped.2020.626182

58. Malviya A, Mishra A. Childhood multisystem inflammatory syndrome: an emerging disease with prominent cardiovascular involvement-a scoping review. SN Compr Clin Med. 2021; 348-59.

59. Porritt RA, Binek A, Paschold L, et al. The autoimmune signature of hyperinflammatory multisystem inflammatory syndrome in children. J Clin Invest. 2021;131(20). doi:10.1172/JCI151520

60. Consiglio CR, Cotugno N, Sardh F, et al. The immunology of multisystem inflammatory syndrome in children with COVID-19. Cell. 2020;183(4):968-981.e7. doi:10.1016/j.cell.2020.09.016

61. Lee PY, Day-Lewis M, Henderson LA, et al. Distinct clinical and immunological features of SARS-CoV-2-induced multisystem inflammatory syndrome in children. J Clin Invest. 2020;130 (11):5942-5950. doi:10.1172/JCI141113

62. Lazarevic I, Pravica V, Miljanovic D, et al. Immune evasion of SARS-CoV-2 emerging variants: what have we learnt so far? Viruses. 2021;13(7):1192. doi:10.3390/v13071192

63. McCormick KD, Jacobs JL, Mellors JW. The emerging plasticity of SARS-CoV-2. Science. 2021;371(6536):1306-1308. doi:10. 1126/science.abg4493

64. Koyama T, Platt D, Parida L. Variant analysis of SARS-CoV-2 genomes. Bull World Health Organ. 2020;98(7):495-504. doi:10.2471/BLT.20.253591

65. Singh J, Samal J, Kumar V, et al. Structure-function analyses of new SARS-CoV-2 variants B.1.1.7, B.1.351 and B.1.1.28.1: clinical, diagnostic, therapeutic and public health implications. Viruses. 2021;13(3):439. doi:10.3390/v13030439

66. Plante JA, Liu Y, Liu J, et al. Spike mutation D614G alters SARS-CoV-2 fitness. Nature. 2021;592(7852):116-121. doi:10. 1038/s41586-020-2895-3

67. Herlihy R, Bamberg W, Burakoff A, et al. Rapid increase in circulation of the SARS-CoV-2 B.1.617.2 (Delta) variant - Mesa County, Colorado, April-June 2021. MMWR Morb Mortal Wkly Rep. 2021;70(32):1084-1087. doi:10.15585/mmwr.mm7032e2

68. Mallapaty S. COVID vaccines slash viral spread - but Delta is an unknown. Nature. 2021;596(7870):17-18. doi:10.1038/d41586021-02054-z

69. Baraniuk C. Covid-19: how effective are vaccines against the delta variant? BMJ. 2021;374:n1960. doi:10.1136/bmj.n1960

70. Ledford H. Should children get COVID vaccines? What the science says. Nature. 2021;595(7869):638-639. doi:10.1038/d4 1586-021-01898-9

71. Irfan O, Li J, Tang K, et al. Risk of infection and transmission of SARS-CoV-2 among children and adolescents in households communities and educational settings: a systematic review and meta-analysis. J Glob Health. 2021;11:05013.

72. Kornitzer J, Johnson J, Yang M, et al. A systematic review of characteristics associated with COVID-19 in children with typical presentation and with multisystem inflammatory syndrome. Int J Environ Res Public Health. 2021;18(16):8269. doi:10. 3390/ijerph18168269 
73. Göktuğ A, Güngör A, Öz FN, et al. Evaluation of epidemiological, demographic, clinical characteristics and laboratory findings of COVID-19 in the pediatric emergency department. J Trop Pediatr. 2021;67(4). doi:10.1093/tropej/ fmab066

74. Belsky JA, Tullius BP, Lamb MG, et al. COVID-19 in immunocompromised patients: a systematic review of cancer, hematopoietic cell and solid organ transplant patients. J Infect. 2021;82 (3):329-338. doi:10.1016/j.jinf.2021.01.022

75. Connelly JA, Chong $\mathrm{H}$, Esbenshade AJ, et al. Impact of COVID-19 on pediatric immunocompromised patients. Pediatr Clin North Am. 2021;68(5):1029-1054. doi:10.1016/j.pcl.2021. 05.007

76. Boronat S. Neurologic care of COVID-19 in children. Front Neurol. 2020;11:613832. doi:10.3389/fneur.2020.613832

77. Raj SL, Vasanthi T, Baineni R, et al. Neurological manifestations of COVID-19 in children. Indian Pediatr. 2020;57(12):11 85-1186. doi:10.1007/s13312-020-2079-0

78. Hoseinyazdi M, Esmaeilian S, Jahankhah R, et al. Clinical, laboratory, and chest CT features of severe versus non-severe pediatric patients with COVID-19 infection among different age groups. BMC Infect Dis. 2021;21(1):560. doi:10.1186/s12879021-06283-5

79. Bustos BR, Jaramillo-Bustamante JC, Vasquez-Hoyos P, et al. Pediatric inflammatory multisystem syndrome associated with SARS-CoV-2: a case series quantitative systematic review. Pediatr Emerg Care. 2021;37(1):44-47. doi:10.1097/PEC.000 0000000002306

80. Lim CC, Saniasiaya J, Kulasegarah J. Croup and COVID-19 in a child: a case report and literature review. BMJ Case Rep. 2021;14(9):e244769. doi:10.1136/bcr-2021-244769

81. Chou J, Platt CD, Habiballah S, et al. Mechanisms underlying genetic susceptibility to multisystem inflammatory syndrome in children (MIS-C). J Allergy Clin Immunol. 2021;148(3):732-738. e1. doi:10.1016/j.jaci.2021.06.024

82. Cui X, Zhang T, Zheng J, et al. Children with coronavirus disease 2019: a review of demographic, clinical, laboratory, and imaging features in pediatric patients. $J$ Med Virol. 2020;92(9):1501-1510. doi: $10.1002 / \mathrm{jmv} .26023$

83. Erat T, Güler Ş. Use of radiological tests in COVID-19 positive child cases: is chest computed tomography necessary? Int J Clin Pract. 2021; 14259 .

84. Foust AM, McAdam AJ, Chu WC, et al. Practical guide for pediatric pulmonologists on imaging management of pediatric patients with COVID-19. Pediatr Pulmonol. 2020;55(9):22 13-2224. doi:10.1002/ppul.24870

85. Islam N, Ebrahimzadeh S, Salameh JP, et al. Thoracic imaging tests for the diagnosis of COVID-19. Cochrane Database Syst Rev. 2021;3:Cd013639.

86. Gregori G, Sacchetti R. Lung ultrasound in outpatient approach to children with suspected COVID 19. Ital J Pediatr. 2020;46 (1):171. doi:10.1186/s13052-020-00938-w

87. Volpicelli G, Gargani L, Perlini S, et al. Lung ultrasound for the early diagnosis of COVID-19 pneumonia: an international multicenter study. Intensive Care Med. 2021;47(4):444-454. doi:10. 1007/s00134-021-06373-7

88. Musolino AM, Boccuzzi E, Supino MC, et al. Point-of-care lung ultrasound in the diagnosis and monitoring of paediatric patients with spontaneous pneumothorax in SARS-CoV-2 infection. J Paediatr Child Health. 2021;57(5):604-606. doi:10.1111/ jpc. 15410

89. Lazzerini M, Sforzi I, Trapani S, et al. Characteristics and risk factors for SARS-CoV-2 in children tested in the early phase of the pandemic: a cross-sectional study, Italy, 23 February to 24 May 2020. Euro Surveill. 2021;26(14). doi:10.2807/15607917.ES.2021.26.14.2001248
90. Qi K, Zeng W, Ye M, et al. Clinical, laboratory, and imaging features of pediatric COVID-19: a systematic review and meta-analysis. Medicine. 2021;100(15):e25230. doi:10.1097/MD. 0000000000025230

91. Chiotos K, Hayes M, Kimberlin DW, et al. Multicenter interim guidance on use of antivirals for children with coronavirus disease 2019/severe acute respiratory syndrome coronavirus 2 . J Pediatric Infect Dis Soc. 2021;10(1):34-48. doi:10.1093/jpids/ piaal15

92. Galindo R, Chow H, Rongkavilit C. COVID-19 in children: clinical manifestations and pharmacologic interventions including vaccine trials. Pediatr Clin North Am. 2021;68(5):961-976. doi:10.1016/j.pcl.2021.05.004

93. Dolinger MT, Person H, Smith R, et al. Pediatric Crohn disease and multisystem inflammatory syndrome in children (MIS-C) and COVID-19 treated with infliximab. J Pediatr Gastroenterol Nutr. 2020;71(2):153-155. doi:10.1097/MPG.0000000000002809

94. Lee J, Lee J, Kim HJ, et al. TMPRSS2 and RNA-dependent RNA polymerase are effective targets of therapeutic intervention for treatment of COVID-19 caused by SARS-CoV-2 variants (B.1.1.7 and B.1.351). Microbiol Spectr. 2021;e0047221.

95. Xia S, Lan Q, Zhu Y, et al. Structural and functional basis for pan-CoV fusion inhibitors against SARS-CoV-2 and its variants with preclinical evaluation. Signal Transduct Target Ther. 2021;6 (1):288. doi:10.1038/s41392-021-00712-2

96. Ren JL, Zhang AH, Wang XJ. Traditional Chinese medicine for COVID-19 treatment. Pharmacol Res. 2020;155:104743. doi:10. 1016/j.phrs.2020.104743

97. Zhao Z, Li Y, Zhou L, et al. Prevention and treatment of COVID-19 using traditional Chinese medicine: a review. Phytomedicine. 2021;85:153308. doi:10.1016/j.phymed.2020. 153308

98. Ren W, Liang P, Ma Y, et al. Research progress of traditional Chinese medicine against COVID-19. Biomed Pharmacother. 2021;137:111310. doi:10.1016/j.biopha.2021.111310

99. Wang WY, Xie Y, Zhou H, et al. Contribution of traditional Chinese medicine to the treatment of COVID-19. Phytomedicine. 2021;85:153279.

100. Li Y, Bi L, Li Y, et al. The effectiveness and safety of traditional Chinese medicine for the treatment of children with COVID-19. Medicine. 2020;99(30):e21247. doi:10.1097/MD.0000000000021 247

101. Cerullo G, Negro M, Parimbelli M, et al. The long history of vitamin C: from prevention of the common cold to potential aid in the treatment of COVID-19. Front Immunol. 2020;11:574029. doi:10.3389/fimmu.2020.574029

102. Panfili FM, Roversi M, D'Argenio P, et al. Possible role of vitamin $\mathrm{D}$ in Covid-19 infection in pediatric population. $J$ Endocrinol Invest. 2021;44(1):27-35. doi:10.1007/s40618-02001327-0

103. Jung LS, Gund TM, Narayan M. Comparison of binding site of remdesivir and its metabolites with NSP12-NSP7-NSP8, and NSP3 of SARS CoV-2 virus and alternative potential drugs for COVID-19 treatment. Protein J. 2020;39(6):619-630. doi:10.10 07/s10930-020-09942-9

104. Wang Y, Zhang Y, Chen X, et al. Evaluating the efficacy and safety of bromhexine hydrochloride tablets in treating pediatric COVID-19: a protocol for meta-analysis and systematic review. Medicine. 2020;99(37):e22114. doi:10.1097/MD.0000000000022114

105. Ensinck G, Gregorio G, Flores RM, et al. [Consensus on treatment of multisystemic inflammatory syndrome associated with COVID-19]. Arch Argent Pediatr. 2021;119(4):S198-S211. Spanish.

106. Dove ML, Jaggi P, Kelleman M, et al. Multisystem inflammatory syndrome in children: survey of protocols for early hospital evaluation and management. $J$ Pediatr. 2021;229:33-40. doi:10.1016/j.jpeds.2020.10.026 
107. Belhadjer Z, Méot M, Bajolle F, et al. Acute heart failure in multisystem inflammatory syndrome in children in the context of global SARS-CoV-2 pandemic. Circulation. 2020;142 (5):429-436. doi:10.1161/CIRCULATIONAHA.120.048360

108. Jonat B, Gorelik M, Boneparth A, et al. Multisystem inflammatory syndrome in children associated with coronavirus disease 2019 in a children's hospital in New York City: patient characteristics and an institutional protocol for evaluation, management, and follow-up. Pediatr Crit Care Med. 2021;22(3):e178-e191. doi:10.1097/PCC.0000000000002598

109. Ghanei M, Solaymani-Dodaran M, Qazvini A, et al. The efficacy of corticosteroids therapy in patients with moderate to severe SARS-CoV-2 infection: a multicenter, randomized, open-label trial. Respir Res. 2021;22(1):245. doi:10.1186/s12931-021-018 33-6

110. Jain MK, Sahu SK, Behera JR, et al. Multisystem inflammatory syndrome in children associated with COVID 19 treated with oral steroid. Indian J Pediatr. 2021;88(1):106. doi:10.1007/s12098020-03497-4

111. Son MBF, Murray N, Friedman K, et al. Multisystem inflammatory syndrome in children - initial therapy and outcomes. $N$ Engl J Med. 2021;385(1):23-34. doi:10.1056/NEJMoa2102 605

112. Belhadjer Z, Auriau J, Méot M, et al. Addition of corticosteroids to immunoglobulins is associated with recovery of cardiac function in multi-inflammatory syndrome in children. Circulation. 2020;142(23):2282-2284. doi:10.1161/CIRCULATIONAHA.12 0.050147
113. Khiali S, Entezari-Maleki T. Therapeutic application of corticosteroids in COVID-19: a focus on optimum dose and duration of therapy. JClin Pharmacol. 2021;61(9):1145-1148. doi:10.1002/jcph.1929

114. National Institute for Health and Care Excellence. Guidelines, in COVID-19 rapid guideline: children and young people who are immunocompromised. National Institute for Health and Care Excellence (NICE); 2020.

115. El Dannan H, Al Hassani M, Ramsi M. Clinical course of COVID-19 among immunocompromised children: a clinical case series. $B M J$ Case Rep. 2020;13(10):e237804. doi:10.1136/bcr-2020-237804

116. Fishman JA, Grossi PA. Novel Coronavirus-19 (COVID-19) in the immunocompromised transplant recipient: \#Flatteningthecurve. $\mathrm{Am}$ J Transplant. 2020;20(7):1765-1767. doi:10.1111/ajt.15890

117. Saeed B. Impact of COVID-19 pandemic on management of pediatric kidney transplant recipients. Exp Clin Transplant. 2021;19(9):894-898. doi:10.6002/ect.2021.0275

118. Bai H, Ji Y, Wang J, et al. Efficacy of human coronavirus immune convalescent plasma for the treatment of Corona virus disease -19 disease in hospitalized children: a protocol for systematic review and meta analysis. Medicine. 2020;99(45):e22017. doi:10.1097/MD.0000000000022017

119. Joyner MJ, Klompas AM, Klassen SA, et al. In reply-how safe is COVID-19 convalescent plasma? Mayo Clin Proc. 2021;96 (8):2281-2282. doi:10.1016/j.mayocp.2021.06.010

120. Tezer H, Bedir Demirdağ T. Novel coronavirus disease (COVID-19) in children. Turk J Med Sci. 2020;50(Si1):592-603. doi:10.3906/sag-2004-174
International Journal of General Medicine

\section{Publish your work in this journal}

The International Journal of General Medicine is an international, peer-reviewed open-access journal that focuses on general and internal medicine, pathogenesis, epidemiology, diagnosis, monitoring and treatment protocols. The journal is characterized by the rapid reporting of reviews, original research and clinical studies across all disease areas. The manuscript management system is completely online and includes a very quick and fair peer-review system, which is all easy to use. Visit http://www.dovepress.com/ testimonials.php to read real quotes from published authors. 